\title{
MESOSCOPIC DERIVATION OF A FUNDAMENTAL DIAGRAM OF ONE-LANE TRAFFIC
}

\author{
MAX-OLIVIER HONGLER AND ROGER FILLIGER
}

\begin{abstract}
We derive from a solvable, discrete velocity model of a nonlinear Boltzmann equation the functional relations between the vehicular density $\rho(x, t)$ and the flux $J(x, t)$ which occurs in a popular traffic model first proposed by Greenshields.
\end{abstract}

\section{INTRODUCTION}

Since the pioneering work of Lighthill and Whitham [1] in the early 50's, a large body of research has been devoted to understand the fundamental principles governing the flow of vehicular traffic. Two complementary approaches can be distinguished:

i) a purely microscopic approach in which the individual vehicular interactions are taken into account (see [2] and the references therein),

ii) a macroscopic approach which is based on fluid dynamical equations describing the behaviour of a compressible fluid (see [3] and the references therein).

The macroscopic description is always based on a continuity equation,

$$
\partial_{t} \rho(x, t)+\partial_{x} J(x, t)=0
$$

and completed by a relation between the current $J(x, t)$ and the vehicle density $\rho(x, t)$, which is known in traffic engineering as the fundamental diagram. This relation contains all the dynamic information specific to a particular macroscopic model. Among the various fundamental diagrams which have been explored (see e.g. $[2,4,5,6])$, a very simple and popular one is the Lighthill-Whitham equation which is characterised by the form:

$$
J(x, t)=j(\rho(x, t))-D \partial_{x} \rho(x, t),
$$

where $D>0$ is a diffusion constant.

Moreover, on the basis of experimental observations, B.D. Greenshields [4] proposed the choice:

$$
j(\rho(x, t))=V_{\max } \rho(x, t)(1-\rho(x, t)),
$$

where the phenomenological parameter $V_{\max }$ is the maximum average speed for $\rho \rightarrow 0$. Eq.(2) together with eq.(3) is known as the improved Greenshields model.

\footnotetext{
Key words and phrases. Nonlinear Boltzmann-equation, Burgers-equation, Fluid-dynamical theories of vehicular traffic.

PACS.45.70.Vn;PACS.89.40.+k;PACS.02.50.Fy

R.F. is partially supported by the Fonds Nationale Suisse pour la recherche scientifique (FNRS) M.O.H. is partially supported by FCT (Portugal).
} 
Besides on its qualitative relevance, it is worth remarking that the improved Greenshields model together with eq.(1) is reduced, via the linear transformation $(x, t) \mapsto$ $(y, \tau):=\left(x-V_{\max } t, t\right)$, to

$$
\partial_{\tau} \rho(y, \tau)-2 V_{\max } \rho(y, \tau) \partial_{y} \rho(y, \tau)=D \partial_{y}^{2} \rho(y, \tau)
$$

which is directly recognised as the viscous form of the famous Burgers' equation. Between the purely microscopic and macroscopic levels of description, it is also interesting to explore an intermediate, mesoscopic, level based on Boltzmann-type kinetic equations $[7,8,9]$. This last approach, which is the one adopted in the present note, provides a deeper understanding of the origin and form of the fundamental diagram. In particular, we shall show how the improved Greenshields model can be obtained by following such an approach. Our derivation is based on the use of a solvable nonlinear discrete velocity Boltzmann equation first introduced by Ruijgrok-Wu in [10].

The paper is organised as follows: In section 1, we briefly review the two-velocity model of the Boltzmann equation introduced in [10]. In section 2, we derive the fundamental diagram equation (2) together with Greenshields' relation eq.(3) by using a Boltzmann-type model similar to the one introduced in section 1. In section 3 , we discuss the probabilistic background of the derivation and the last section is devoted to some conclusions.

\section{The Ruijgrok-Wu model and its connection to the Burgers' EQUATION}

The one-dimensional, nonlinear two-velocity Boltzmann model studied by Th.W Ruijgrok and T.T. Wu in [10], to which we shall refer as the RW-model, is of the form:

$$
\left\{\begin{array}{l}
\left(\partial_{t}+v_{1} \partial_{x}\right) \hat{f}=\mu \hat{f} \hat{g}-\alpha \hat{f}+\beta \hat{g} \\
\left(\partial_{t}+v_{2} \partial_{x}\right) \hat{g}=-\mu \hat{f} \hat{g}+\alpha \hat{f}-\beta \hat{g}
\end{array}\right.
$$

where $(x, t) \in \mathbb{R} \times \mathbb{R}^{+}, \hat{f}:=\hat{f}(x, t)$ and $\hat{g}:=\hat{g}(x, t)$ are the distribution functions of vehicles with velocities $v_{1}$ and $v_{2}$ respectively and where $\alpha, \beta$ and $\mu$ are positive constants. As explained in [10], the model takes into account three velocity exchange mechanisms contained on the right hand side of eqs.(5), namely:

(a) a binary collision of the form $\left(v_{1}, v_{2}\right) \stackrel{\mu}{\longrightarrow}\left(v_{1}, v_{1}\right)$, modelling a transition from $\left(v_{1}, v_{2}\right)$ to $\left(v_{1}, v_{1}\right)$ with intensity $\mu$,

(b) a spontaneous transition of the form $\left(v_{1}\right) \stackrel{\alpha}{\longrightarrow}\left(v_{2}\right), \alpha$ being the rate of transitions, and

(c) a spontaneous transition of the form $\left(v_{2}\right) \stackrel{\beta}{\longrightarrow}\left(v_{1}\right), \beta$ being the rate of transitions.

Note that the absence of inverse collision of type (a) indicates violation of the detailed balance, which is an intrinsic feature of vehicular traffic [11]. In fact, a fast driver with speed $v_{2}$, say, behind a slow one with velocity $v_{1}<v_{2}$ is slowed down according to (a). 
Suppose now (without loss of generality as it will be seen) that $v_{1}=-v_{2}=: \gamma$ and that the densities are given by a logarithmic transformation of the form:

$$
\left\{\begin{array}{l}
\hat{f}(x, t)=\frac{1}{\mu}\left(\partial_{t}-\gamma \partial_{x}\right)(\ln (u(x, t))+a x+b t) \\
\hat{g}(x, t)=-\frac{1}{\mu}\left(\partial_{t}+\gamma \partial_{x}\right)(\ln (u(x, t))+a x+b t)
\end{array}\right.
$$

for a strictly positive function $u:=u(x, t)>0$ and two constants $a$ and $b$.

With these assumptions eqs.(5) simplifies to the linear hyperbolic PDE

(7) $\partial_{t}^{2} u+(\alpha+\beta+2 b) \partial_{t} u=\gamma^{2} \partial_{x}^{2} u+\gamma(\alpha-\beta+2 \gamma a) \partial_{x} u+u \cdot\left((\gamma a)^{2}+\gamma a(\alpha-\beta)-b^{2}-b(\alpha+\beta)\right)$.

By the ad-hoc choice of the constants $a:=\frac{\beta-\alpha}{2 \gamma}$ and $b:=-\frac{\alpha+\beta}{2}+\sqrt{\frac{\alpha^{2}+\beta^{2}}{2}}$, eq.(7) reduces to:

$$
\partial_{t}^{2} u+\sqrt{2\left(\alpha^{2}+\beta^{2}\right)} \partial_{t} u=\gamma^{2} \partial_{x}^{2} u
$$

which is the famous telegraphist equation.

The logarithmic transform (L-T) which reduces the non-linear eqs. (5) to the linear telegraphist equation (8) plays a role similar to the well-known Hopf-Cole transformation (H-C), reducing the Burgers' equation to the (linear) heat equation. This observation, thoroughly discussed in [12], and the central role of the Burgers' equation in Greenshields' model, leads us to emphasize the importance of the RW-model in the traffic context.

\section{Derivation of Greenshields' Model}

In this section we show how the improved Greenshields' model:

$$
J(x, t)=V_{\max } \cdot \rho(x, t)(1-\rho(x, t))-D \partial_{x} \rho(x, t)
$$

can be mesoscopically derived. To this aim, we first link the coordinates to the center of inertia:

$$
(x, t) \mapsto(x-\bar{v} t, t), \quad \bar{v}:=\frac{v_{1}+v_{2}}{2} .
$$

In this coordinates, the velocities of the vehicles are transformed as

$$
v_{1} \mapsto-v_{0}, \quad v_{2} \mapsto v_{0},
$$

where

$$
v_{0}:=\frac{v_{2}-v_{1}}{2} .
$$

The second step is a diffusive re-scaling of the coordinates of the form

$$
y:=c(x-\bar{v} t), \quad \tau:=c^{2} t,
$$

which is accompanied by the normalisation transformation

$$
\hat{f} \mapsto f=\hat{f} / c, \quad \hat{g} \mapsto g=\hat{g} / c,
$$

where $c$ is a dimensionless scaling parameter. The diffusive limit $c \rightarrow 0$ (see Ch. VIII in [13]) corresponds to the transition from a kinetic to a macroscopic description of the traffic. With the coordinates given by (11) and the diffusive re-scaling (13, $14)$, the system (5) reads:

$$
\left\{\begin{array}{l}
\left(\partial_{\tau}-\frac{v_{0}}{c} \partial_{y}\right) f=\frac{\mu}{c} f g-\frac{\alpha}{c^{2}} f+\frac{\beta}{c^{2}} g \\
\left(\partial_{\tau}+\frac{v_{0}}{c} \partial_{y}\right) g=-\frac{\mu}{c} f g+\frac{\alpha}{c^{2}} f-\frac{\beta}{c^{2}} g .
\end{array}\right.
$$


The macroscopic variables for the system (15) are the vehicle density $\rho:=\rho(y, \tau)$ and the flux $J:=J(y, \tau)$ respectively given by:

$$
\begin{aligned}
\rho(y, \tau) & =f(x(y, \tau), t(y, \tau))+g(x(y, \tau), t(y, \tau)) \\
& =\frac{1}{c}(\hat{f}(x(y, \tau), t(y, \tau))+\hat{g}(x(y, \tau), t(y, \tau))), \\
J(y, \tau) & =\frac{v_{0}}{c}(g(x(y, \tau), t(y, \tau))-f(x(y, \tau), t(y, \tau))) \\
& =\frac{v_{0}}{c^{2}}(\hat{g}(x(y, \tau), t(y, \tau))-\hat{f}(x(y, \tau), t(y, \tau))) .
\end{aligned}
$$

By addition of both equations in (15), we immediately have the continuity equation

$$
\partial_{\tau} \rho+\partial_{y} J=0 .
$$

From eqs.(16) and (17), we have:

$$
\left\{\begin{array}{l}
f=\frac{1}{2 v_{0}}\left(v_{0} \rho-c J\right) \\
g=\frac{1}{2 v_{0}}\left(v_{0} \rho+c J\right) .
\end{array}\right.
$$

Introducing the linear combination of the two equations in the system (15):

$$
-v_{0} \cdot\left(\partial_{\tau}-\frac{v_{0}}{c} \partial_{y}\right) f+v_{0} \cdot\left(\partial_{\tau}+\frac{v_{0}}{c} \partial_{y}\right) g=2 v_{0}\left(-\frac{\mu}{c} f g+\frac{\alpha}{c^{2}} f-\frac{\beta}{c^{2}} g\right)
$$

and performing a few elementary manipulations of eqs.(19), we end up with:

$$
\frac{c^{2}}{v_{0}^{2}} \partial_{\tau} J+\partial_{y} \rho=\rho\left(\frac{\alpha-\beta}{c v_{0}}-\frac{\mu}{2 v_{0}} \rho\right)+J\left(\frac{\mu c^{2}}{2 v_{0}^{3}} J-\frac{\alpha+\beta}{v_{0}^{2}}\right) .
$$

To explicitly connect the RW-model with Greenshields equation (9) we introduce the relation:

$$
\alpha=\beta+\frac{\mu}{2} c
$$

and the definitions

$$
D:=\frac{v_{0}^{2}}{\alpha+\beta}, \quad V_{\max }:=\frac{\mu}{2(\alpha+\beta)} v_{0} .
$$

With these definitions which we discuss below, eq.(21) reduces in the diffusive limit, $c \rightarrow 0$, to Greenshields' flux relation:

$$
J=V_{\max } \cdot \rho(1-\rho)-D \partial_{y} \rho .
$$

\section{Discussion}

At this point, it is worth-while to comment the physical content of eq.(22). First we see from the fact that the collisions $\left(v_{1}, v_{2}\right) \stackrel{\mu}{\longrightarrow}\left(v_{1}, v_{1}\right)$ steadily increases the density of particles having velocity $v_{1}$, the only possibility to reach an equilibrium is to have a transition imbalance $\alpha>\beta$. The derivation of (22) shows that for an equilibrium to exist, the difference of transition rats $\alpha-\beta$ should read as $c \mu / 2$. Note that the constant $D$ as given in (23) is consistent with the diffusion constant 
$\bar{D}$ of the telegraphist equation. In fact, when replacing in (8) $\alpha$ by $\frac{\alpha}{c^{2}}, \beta$ by $\frac{\beta}{c^{2}}$, and $\gamma$ by $\frac{v_{0}}{c}$ one finds the telegraphist equation associated to (15):

$$
\partial_{t} u=\underbrace{\frac{v_{0}^{2}}{\sqrt{2\left(\alpha^{2}+\beta^{2}\right)}}}_{=: \bar{D}} \partial_{x}^{2} u-\frac{c^{2}}{\sqrt{2\left(\alpha^{2}+\beta^{2}\right)}} \partial_{t}^{2} u,
$$

and using (22), we see that $\lim _{c \rightarrow 0} \bar{D}=\lim _{c \rightarrow 0} D=v_{o}^{2} /(2 \alpha)$.

Let as now also examine the diffusive limit $c \rightarrow 0$. For this, consider the piecewise deterministic process solving the stochastic differential equation:

$$
\mathrm{d} X_{\gamma}(t):=\gamma I\left(\gamma^{2} t\right) \mathrm{d} t
$$

where $\gamma:=\frac{v_{0}}{c}$ and $\gamma I\left(\gamma^{2} t\right)$ is a random telegraph process (i.e. a continuous time two states Markov chain) which takes on values in $\{-\gamma,+\gamma\}$ and whose transition rate is given by $\sqrt{2\left(\alpha^{2}+\beta^{2}\right)} /\left(2 c^{2}\right)$. The Master equation that can be associated with the Markov process $\left(X_{\gamma}(t), \gamma I\left(\gamma^{2} t\right)\right)$ has the form

$$
\frac{\mathrm{d} T_{t} \phi}{\mathrm{d} t}=G T_{t} \phi
$$

where the operator $T_{t}$ is the stochastic semigroup associated with the Markov process $\left(X_{\gamma}(t), \gamma I\left(\gamma^{2} t\right)\right)$ acting on locally bounded and measurable functions $\phi$ defined on $\mathbb{R} \times\{-\gamma, \gamma\}$. It is defined as

$$
T_{t} \phi(x, y)=\mathbb{E}_{x, y}\left[\phi\left(X_{\gamma}(t), \gamma I\left(\gamma^{2} t\right)\right)\right], \quad(x, y) \in \mathbb{R} \times\{-\gamma, \gamma\},
$$

where $\mathbb{E}_{x, y}$ is the expectation conditioned on the initial values $\left(X_{\gamma}(0), \gamma I(0)\right)=$ $(x, y)$. The infinitesimal generator $G=\left.\frac{\mathrm{d} T_{t} \phi}{\mathrm{d} t}\right|_{t=0}$ of the semigroup $T_{t}$ is given by:

$$
G=\left(\begin{array}{cc}
-\gamma \partial_{x} & 0 \\
0 & \gamma \partial_{x}
\end{array}\right)+\sqrt{2\left(\alpha^{2}+\beta^{2}\right)} /\left(2 c^{2}\right)\left(\begin{array}{cc}
-1 & 1 \\
1 & -1
\end{array}\right) .
$$

These facts discussed in [14], can be used to determine the system of backward equations describing the Markov process which reads:

$$
\frac{\partial}{\partial_{t}}\left(\begin{array}{l}
u_{-}(x, t) \\
u_{+}(x, t)
\end{array}\right)=G\left(\begin{array}{l}
u_{-}(x, t) \\
u_{+}(x, t)
\end{array}\right)
$$

where $u_{-}(x, t):=T_{t} \phi(x,-\gamma)$ and $u_{+}(x, t):=T_{t} \phi(x, \gamma)$ for $\phi$ of class $\mathcal{C}^{1}$. Applying the operators $\partial_{x}, \partial_{t}$ to $(30)$, it is seen that both field components $u_{-}$and $u_{+}$satisfy the telegraphist equation (25). This probabilistic connection between eq.(25) and stochastic processes was first noticed by [15] and [16].

Due to the central limit theorem (CLT), the random telegraph process defined in (26) converges for $c \rightarrow 0$ to a Gaussian white noise and in this limit we can write (26) as:

$$
\mathrm{d} X_{t}:=\sqrt{D} \mathrm{~d} W_{t}
$$

with $W_{t}$ beeing the standard Brownian motion [17].

The Chapman-Kolmogorov equation associated with the process (31) is the heat equation. Moreover, both the heat equation and the telegraphist equation are equivalent, up to nonlinear transformations (L-T) and (H-C), to the Burgers' and the RW-model equations, respectively. This yields the global picture given in the following table: 


\begin{tabular}{|c|c|c|c|c|}
\hline RW-model & $\stackrel{L-T}{\longrightarrow}$ & Telegraphist equation & $\longleftrightarrow$ & telegraph process \\
\hline$\downarrow$ & & $\downarrow c \rightarrow 0$ & & $\downarrow$ CLT \\
\hline Burgers' equation & $\stackrel{H-C}{\longrightarrow}$ & Heat equation & $\longleftrightarrow$ & white noise \\
\hline
\end{tabular}

The previous analysis clearly shows that the improved Greenshields' model eq.(3) follows from the mesoscopic model of vehicular traffic given in eqs.(5) and can be understood from a central limit procedure.

It is interesting to remark that a few mesoscopic models of Boltzmann type has been derived from microscopic considerations. For example De Masi and Presutti derive the Carleman equation - a special case of a discrete Boltzmann equation from a microscopic model where two types of particles evolve on the discrete torus according to independent asymmetric random walks [18]. Rezakhanlou derived discrete Boltzmann equations from microscopic models [19]. In these models particles (vehicles) travel as independent random walks and collide stochastically. The conservation of momentum however - not present in the RW-model - is supposed to hold microscopically. As far as we know, the RW-model has not yet been derived from a microscopic model.

\section{Conclusion}

In this note, we have shown how the single-lane traffic model of Lighthill and Whitham with the Greenshields flux assumption can be derived from a simple and exactly solvable mesoscopic model. This model is isomorphic to a discrete twovelocity Boltzmann-type equation (i.e. the RW-model). Among the basic features of the RW-model, the absence of detailed balance is particularly well suited to model traffic problems. As the RW-model can be seen as an exactly solvable generalisation of the Burgers' equation, it offers a simple and relevant model to be used in traffic theoretical contexts.

\section{ACKNOWLEDGEMENTS}

We are grateful to the referee for most helpful remarks and suggestions.

\section{REFERENCES}

[1] M.J. Lighthill and G.B. Whitham. On kinematic waves II.: A theory of traffic flow on long crowded roads. Proceedings of the Royal Society of London A, 229:317-345, 1955.

[2] D. Chowdhury, L. Santen, and A. Schadschneider. Statistical physics of vehicular traffic and some related systems. Physics Reports, 329:199-329, 2000.

[3] D. Helbing. Traffic and related self-driven many-particle systems. Reviews of Modern Physics, 73 (4):1067-1141, 2001.

[4] B.D. Greenshields. A study of traffic capacity. Proceedings of the Highway Research Board, 14:448-477, 1935.

[5] R.D. Kühne and M.B. Rödiger. Macroscopic simulation model for freeway traffic with jams and stop-start waves. B.L. Nelson, W.D. Kelton, and G.M. Clark, eds. Proceedings of the 1991 Winter Simulation Conference, Phoenix, Arizona., p. 762, 1991.

[6] B.S. Kerner and P. Konhäuser. Cluster effect in initially homogenous traffic flow. Physical Review E, 48:2335-2338, 1993. 
[7] P. Nelson. A kinetic model of vehicular traffic and its associated bimodal equilibrium solutions. Transport Theory and Statistical Physics, 24:383-409, 1995.

[8] S.L. Paveri-Fontana. On Boltzmann-like treatments for traffic flow. Transportation Research, 9:225-235, 1975.

[9] I. Prigogine and R. Herman. Kinetic Theory of Vehicular Traffic. American Elsevier Publishing Co., 1971.

[10] Th.W. Ruijgrok and T.T. Wu. A completely solvable model of the nonlinear Boltzmann equation. Physica, 113 A:401-416, 1982.

[11] B. Derrida. An exactly soluble non-equilibrium system: the asymmetric simple exclusion process. Physics Reports, 301:65-83, 1998.

[12] M.O. Hongler and L. Streit. A probabilistic connection between the Burgers' and a discrete Boltzmann equation. Europhysics Letters, 12(3):193-197, 1990.

[13] N.G. van Kampen. Stochastic Processes in Physics and Chemistry, (see Ch. VIII and especially p.216). North-Holland, 1981.

[14] M.A. Pinsky. Lectures on Random Evolution, pp.3-8. World Scientific Publishing, 1991.

[15] S. Goldstein. On diffusion by discontinuous movements, and on the telegraph equation. Quart. J. Mech. Appl. Math., 4:129-156, 1951.

[16] M. Kac. A stochastic model related to the telegrapher's equation. Rocky Mt. J. Math., 4:497509, 1974.

[17] C.W. Gardiner. Handbook of Stochastic Methods, p.210. Springer-Verlag, 1985.

[18] A. De Masi and E. Presutti. Mathematical methods for hydrodynamic limits. Lecture Notes in Mathematics 1501, Springer-Verlag, 1991.

[19] F. Rezakhanlou. Propagation of chaos for particle systems associated with discrete Boltzmann equation. Stochastic Processes and their Appl., 64:55-72, 1996.

Permanent address, Département de Microtechnique (DMT), Institut de Production MiCROtechnique (IPM), E.P.F.L., CH-1015 LAUSANNE.

M.O.H. is also with, Centro de Ciencias Matematicas (CCM), Universidade da Madeira, P-9000 Funchal (Portugal).

E-mail address: max.hongler@epfl.ch roger.filliger@epfl.ch 